\title{
Clinical Outcomes of an All-Arthroscopic Technique for Single-Stage Autologous Matrix-Induced Chondrogenesis in the Treatment of Articular Cartilage Lesions of the Knee
}

\author{
Andrew Chia Chen Chou, M.D., M.R.C.S., and \\ Denny Tjiauw Tjoen Lie, M.B.B.S., F.R.C.S., F.A.M.S.
}

\begin{abstract}
Purpose: The purpose of this study was to determine the clinical efficacy of an all-arthroscopic approach to autologous matrix-induced chondrogenesis (AMIC) for patients with articular cartilage lesions of the knee joint. We hypothesize that an all-arthroscopic, single-stage AMIC using a hyaluron-based cell-free scaffold improves the postoperative clinical scores for patients with isolated articular cartilage lesions of the knee in the early follow-up period. Methods: All patients with focal osteochondral lesions of the knee treated with AMIC at our institution from November 2013 to January 2018 were included for analysis. Demographic information, baseline clinical characteristics, perioperative imaging, and follow-up International Knee Documentation Committee (IKDC) scores at 6 and 24 months postoperatively were collected. One-way analysis of variance (ANOVA) with a Bonferroni correction was used to assess for improvement before and after surgery, with statistical significance defined as $p<.05$. Results: A total of 22 patients met the inclusion criteria and were analyzed for this study. Articular cartilage lesions were most commonly found at the center of the medial and lateral femoral condyles. Statistically significant improvements in IKDC scores were seen at the 6- and 24-month follow-up periods $(p<.05)$. Conclusion: An all-arthroscopic technique for single-stage autologous matrix-induced chondrogenesis demonstrates significant early clinical improvement for the treatment of articular cartilage lesions of the knee. Level of Evidence: 4
\end{abstract}

A s human articular cartilage has limited intrinsic healing capacity, focal articular cartilage defects of the knee do not heal independently and frequently predispose patients to early osteoarthritis, which can lead to persistent knee pain, functional disability, and reduced quality of life. ${ }^{1-5}$ Although multiple surgical options, such as arthroscopic debridement,

From the Department of Orthopaedic Surgery, Singapore General Hospital, Singapore.

The authors report that they have no conflicts of interest in the authorship and publication of this article. Full ICMJE author disclosure forms are available for this article online, as supplementary material.

Received December 5, 2019; accepted May 13, 2020.

Address correspondence to Andrew Chou, M.D., M.R.C.S., Department of Orthopaedic Surgery, the Academia, 20 College Road, Singapore 169856; +65 8818 3934.E-mail: andrew.chou@alumni.stanford.edu

(C) 2020 THE AUTHORS. Published by Elsevier Inc. on behalf of the Arthroscopy Association of North America. This is an open access article under the CC BY-NC-ND license (http://creativecommons.org/licenses/by-nc-nd/4.0/). 2666-061X/191471

https://doi.org/10.1016/j.asmr.2020.05.006 chondroplasty, osteochondral autografts, and osteoarticular transfer systems, have been proposed as treatment modalities for articular cartilage defects of the knee, no individual technique is able to induce articular cartilage regeneration with both hyaline-like cartilage and seamless integration into the surrounding cartilage and underlying bone. ${ }^{3,6}$

Bone marrow stimulation techniques, such as microfracture, were first developed in the 1950s by Pridie and Steadman with the aim of inducing subchondral bone bleeding, thereby releasing multipotent mesenchymal stem cells and growth factors into the defect site, resulting in the formation of a clot and eventual repair of the cartilage defect with fibrous and hyaline-like cartilage..$^{7-10}$ Particularly in comparison to other techniques such as osteochondral grafting or transfer systems, microfracture avoids donor site morbidity and the need for multiple surgeries. ${ }^{6,11}$ However, although it provides robust clinical results for smaller defects at a lower cost compared with osteoarticular allografts or staged osteochondral 
transplantation, the microfracture technique is limited because the resulting clot tends to produce a mix of fibrous and hyaline-like cartilage, thus having a poorer capacity to withstand repetitive load bearing. $5,6,9,12,13$

With the advent of cell-free biological scaffolds, autologous matrix-induced chondrogenesis (AMIC) has been introduced as an innovative, single-stage solution to improve the efficacy of microfracture. ${ }^{3,5,11,12}$ Via placement of biological scaffolds composed of porcine collagen matrix, polyglycolic acid, or hyaluronic acid over the cartilage defect after microfracture, AMIC provides a mechanically stable structure for subchondral blood to soak into. ${ }^{4,11,14}$ The matrix thereby acts as a biocompatible, temporary scaffold that concentrates the mesenchymal stem cells and growth factors in place, thereby concentrating them only over the defect and not throughout the joint. ${ }^{4,11,14}$ However, placement of the scaffolds can be technically challenging, as the matrices become soft and adherent upon contact with fluid or blood, and visualization can be limited through an arthroscopic camera. Thus, authors often describe the need to extend the arthroscopic portals or perform a mini-arthrotomy to facilitate placement, which may increase wound-related complications and delay recovery. ${ }^{5,15}$

As the popularity of cell-free biological scaffolds and the AMIC technique grows, there is a need to assess the clinical efficacy of an all-arthroscopic AMIC technique in the treatment of focal articular cartilage defects of the knee. Currently, limited literature is available that examines the early and medium-term outcomes of focal cartilage injuries of the knee treated with microfracture in conjunction with a cell-free hyaluron-based scaffold. The purpose of this study was to determine the clinical efficacy of an all-arthroscopic approach to AMIC for patients with articular cartilage lesions of the knee joint.

\section{Methods}

\section{Patient Recruitment}

This study analyzed the prospectively collected data of all patients who underwent AMIC using a cell-free hyaluron-based scaffold at our institution between November 2013 and January 2018. Approval was granted by our tertiary center's institutional review board. The inclusion criteria were patients aged 18 to 65 years with symptomatic articular cartilage defects of the medial femoral condyle, lateral femoral condyle, or trochlea, identified as International Cartilage Repair Society (ICRS) grade III and higher on preoperative magnetic resonance imaging (MRI) , <20 $\mathrm{mm}$ in diameter, who underwent microfracture with a hyaluron-based cell-free scaffold, and had $\geq 2$ years of follow-up data available. Patients who were $<18$ or $>65$ years old; had a known history of autoimmune or inflammatory arthritis, previous intraarticular fracture, previous total meniscectomy, or instability of the knee; who underwent concurrent ligament reconstruction; had defects $>20 \mathrm{~mm}$ in diameter; or had incomplete or insufficient follow-up data were excluded from the study. The study criteria are shown in Table 1.

\section{Data Collection}

The medical records, radiographic imaging, and surgical reports of all patients who met the inclusion criteria were collected and reviewed by a sports medicine fellowship-trained orthopaedic surgeon. Clinical data collected included baseline demographics, including age, sex, ethnicity, weight, height, body mass index (BMI), the affected knee, and any concurrent injuries. Surgical reports were reviewed to confirm the procedure done and postoperative rehabilitation plan. Patients were assessed preoperatively, 6 months postoperatively, and 2 years postoperatively using the International Knee Documentation Committee (IKDC) questionnaire, a validated questionnaire that assesses patients on 3 domains, symptoms, sports activity, and knee function, to provide an overall knee function score. ${ }^{16,17}$ Patients were also assessed for postoperative complications, defined as skin or knee infections, skin dehiscence, iatrogenic fracture, hemarthrosis, or persistent pain postoperatively. All pre- and postoperative clinical outcome evaluations were performed by trained physiotherapists at our orthopaedic diagnostic center, an independent unit that tracks clinical outcomes and postoperative functional assessments for our department's patients.

Table 1. Study inclusion and exclusion criteria.

\begin{tabular}{ll}
\hline \multicolumn{1}{c}{ Inclusion Criteria } & \multicolumn{1}{c}{ Exclusion Criteria } \\
\hline Age $>18$ and $<65$ years & Age $<18$ or $>65$ years \\
Symptomatic articular & Known history of \\
cartilage defect, ICRS grade & autoimmune or \\
III and above, and $<20 \mathrm{~mm}$ & inflammatory arthritis, \\
in diameter & diffuse osteoarthritis, \\
& instability of the knee, prior \\
& intraarticular fracture, \\
& previous total \\
& meniscectomy, articular \\
& cartilage defect $>20$ mm in \\
diameter \\
Treated with microfracture & Underwent concurrent \\
with a hyaluron-based cell- & ligamentous reconstruction \\
free scaffold & \\
Preoperative MRI available & Incomplete imaging data \\
$>2$ years of follow-up data & Insufficient or lost to follow- \\
available & up \\
\hline
\end{tabular}

The study's inclusion and exclusion criteria were designed to select for patients with symptomatic articular cartilage lesions $<20 \mathrm{~mm}$ in diameter treated with AMIC using a cell-free hyaluron-based scaffold with both a preoperative MRI and adequate postoperative follow-up. Abbreviations: AMIC, autologous matrix-induced chondrogenesis; ICRS, International Cartilage Repair Society. 
Preoperative MRIs and operative reports were reviewed to better characterize the articular cartilage lesions in terms of location, size in millimeters, and ICRS grade. If intraoperative findings differed from preoperative MRI images, measurements and photos taken via arthroscopy were used instead, as direct visualization of lesions was likely to be more accurate. To better define the location of these defects, the femoral condyles were further numbered and defined by a $3 \times 3$ grid, and the femoral trochlea was divided into thirds (medial, central, and lateral) as per validated ICRS grid map 18. Figure 1 illustrates the grid distribution on the medial femoral condyle $(1 A)$, lateral femoral condyle $(1 B)$, and trochlea $(1 C)$.

\section{Surgical Technique}

All surgical procedures were performed by a sportstrained surgeon on lesions that were ICRS grade III and higher. As previously described by Benthien and Behrens, the surgery began with a routine diagnostic arthroscopy under general anesthesia and tourniquet to confirm preoperative MRI findings and characterize the size and location of the articular cartilage lesion. ${ }^{11}$ After the defect was measured and photographed, the lesion was debrided with an arthroscopic shaver until a stable shoulder surrounded the defect. If concurrent injuries, such as meniscal tears, were identified on preoperative MRI scans or intraoperatively, concurrent procedures, such as meniscus repairs or meniscectomies, were performed before proceeding with the microfracture.

Microfracture was performed using either handheld awls or 1.1-mm Kirschner wires drilled at regular 5-mm intervals, as shown in Figure 2A. Based on measurements made arthroscopically using an arthroscopic probe, a hyaluron-based cell-free scaffold (Hyalofast; Anika Therapeutics, Bedford, MA) was trimmed to match the size of the defect. To facilitate application of the matrix and avoid wetting before contact with the clot, the matrix was inserted with the guidance of makeshift cannula. Such a cannula can be created by removing the plunger of a simple 5-mL plastic syringe and cutting off the tip and distal end of the barrel at an oblique angle, as shown in Figure 2B. Under dry arthroscopy, the cannula was inserted through the arthroscopic ports and slotted directly over the articular cartilage defect, as shown in Figure 2C, allowing for AMIC to be performed entirely arthroscopically, after which the matrix is applied through the cannula, thus allowing it to become wet only after contact with the clot.

Whereas other authors have advised the use of sutures or resorbable pins to stabilize the matrix, such fixation methods can induce local damage similar to early osteoarthritis and were not used in our study. ${ }^{12}$ After the scaffold took on a soft, conformable, adherent texture after coming in contact with blood, the matrix was conformed to the size and shape of the defect using the syringe plunger as a trocar. Multiple scaffold pieces were placed through the cannula if 1 piece of scaffold was insufficient. The scaffold was subsequently allowed to stabilize for $\geq 15$ minutes and was checked for stability in flexion and extension, as shown in Figure 2D, before wound closure and dressing.

\section{Postoperative Rehabilitation Protocol}

The goal of postoperative rehabilitation is to allow for graduated return to weightbearing without destabilizing the hyaluron-based cell-free scaffold by subjecting it to unnecessary compressive or shearing forces. Therefore, weightbearing and immobilization were dependent on the location of the articular cartilage defect. If a defect was located over a weightbearing surface such as the medial or lateral femoral condyles, patients were advised to immobilize the knee in extension with
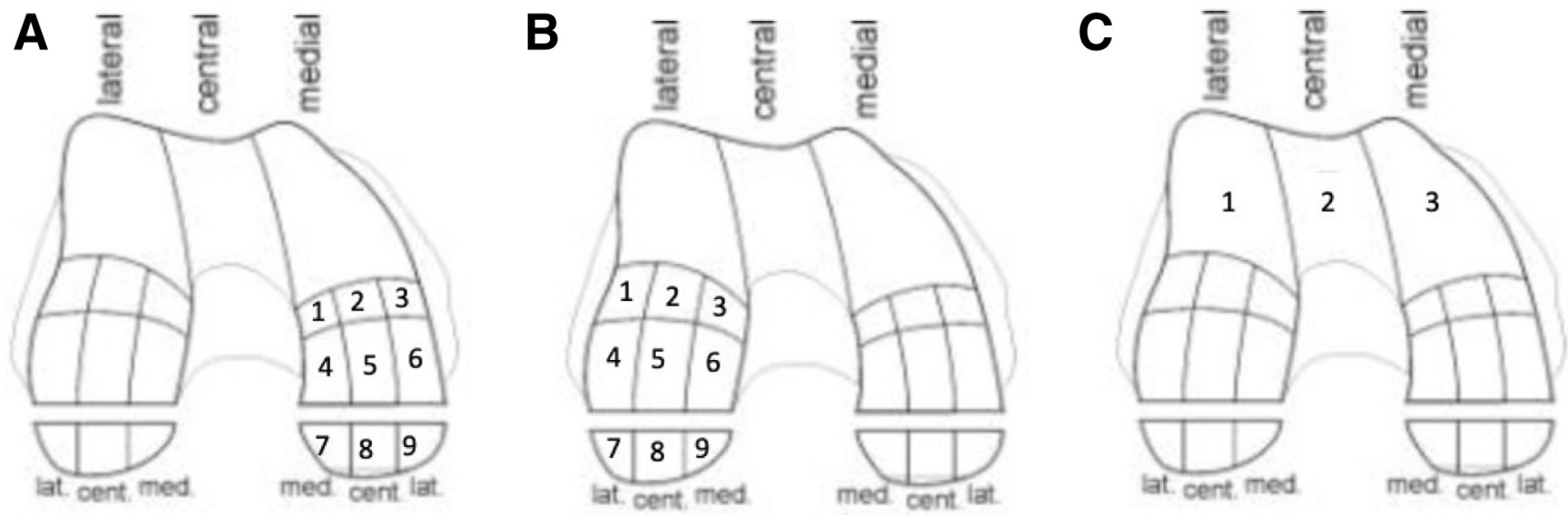

Figure 1. Grid distribution for the medial femoral condyle (A), lateral femoral condyle (B), and femoral trochlea (C). To better characterize the location where articular cartilage defects were occurring, the femoral condyles were divided into $3 \times 3$ grids, and the femoral trochlea was divided into thirds as per International Cartilage Repair Society (ICRS) grid map 18. 


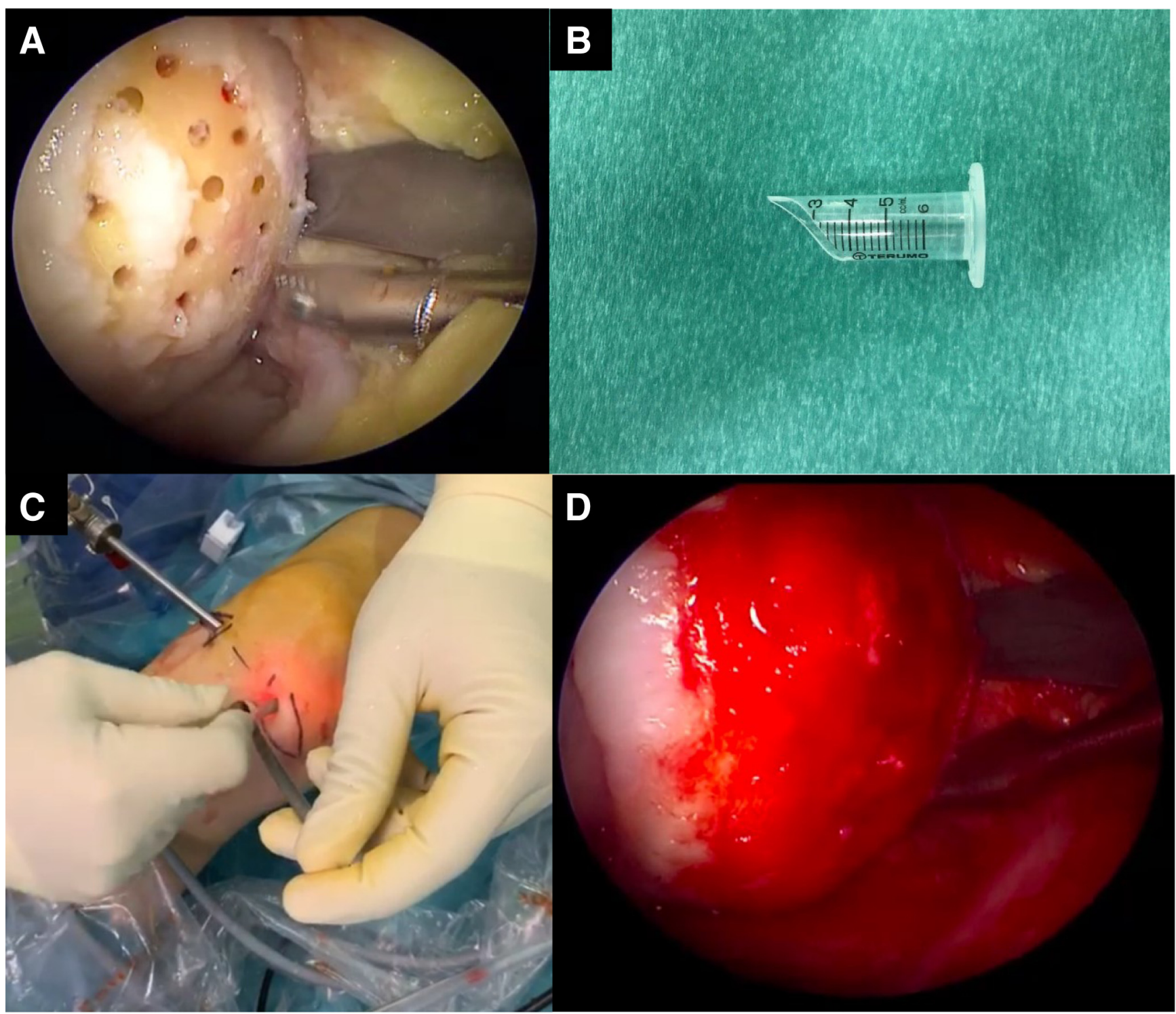

Figure 2. All-arthroscopic technique for autologous matrix-induced chondrogenesis. (A) Microfracture of an osteochondral defect in the lateral femoral condyle of the right knee. After debriding away the defect's edges into a stable shoulder, microfracture is performed at $5 \mathrm{~mm}$ intervals using an awl or K-wire. (B) Fashioning a cannula to facilitate insertion. Cutting a simple plastic syringe at an oblique angle allows for insertion of the Hyalofast scaffold into the knee without getting the scaffold wet prematurely. (C) Inserting the cannula through an arthroscopic port. Using a standard 5-mL syringe allows for insertion through existing arthroscopic portals due to the small size of the cannula. (D) Application of Hyalofast. The Hyalofast scaffold is directly applied onto the defect and becomes conformable and adherent upon contact with the subchondral blood, thereby facilitating application.

non-weightbearing for 4 weeks, followed by partial weightbearing with increasing range of motion for 4 weeks, followed by full weightbearing with a full range of activity. If a defect was located over the trochlea or patella, patients were allowed partial weightbearing with the knee locked in extension for 4 weeks, followed by full weightbearing with full range of motion afterward. As in analogous rehabilitation protocols for cartilage repair techniques, patients were allowed to return to low-impact activities only after 6 months and to sports only after 1 year.

\section{Statistical Analysis}

Statistical Package for the Social Sciences (SPSS) ${ }^{\circledR}$ version 24 (IBM Corp., Armonk, NY) was used for all statistical analyses. Results are presented as means with standard deviations and percentages of the total number of knees in the entire patient group. One-way analysis of variance (ANOVA) with a Bonferroni correction was used to compare baseline IKDC scores with those postoperatively to assess for significant differences between scores. Statistical significance was defined as $p<.05$ 


\section{Results}

\section{Demographic Data}

Of 38 patients who underwent the procedure at our institution in the specified time frame, a total of 22 knees in 22 patients fit the inclusion criteria and were analyzed in this study, with a follow-up rate of $57.9 \%$. Basic demographic information including age, sex, ethnicity, height, weight, BMI, and concurrent injuries are detailed in Table 2. Of the 22 knees analyzed in this study, 19 had concurrent injuries $(86.4 \%)$, and 11 $(50 \%)$ underwent a concurrent arthroscopic procedure in the same setting, usually a meniscus repair, debridement, or meniscectomy.

\section{Characteristics of Articular Cartilage Lesions}

Descriptive data regarding the articular cartilage lesions are summarized in Table 3.

\section{Postoperative Outcomes}

Mean IKDC scores preoperatively were $33.5 \pm 19.08$, $54.58 \pm 17.46$ at 6 months postoperatively, and $66.19 \pm 19.73$ at 12 months. Repeated measures ANOVA conducted between preoperative and 6-month postoperative IKDC scores showed a mean difference of 21.1 (95\% confidence interval [CI] 7.18 to 34.96), which was statistically significant $(p<.01)$. Similarly, a repeated measures ANOVA between 6 months and 2 years postoperatively showed a mean difference of 10.7 (95\% CI 3.26 to 26.49 ), which was statistically significant $(p<.01)$. Mean scores improved by $65.6 \%$ at the 6-month mark and $97.6 \%$ by the 2 -year mark compared with before surgery. No patients were

Table 2. Patient demographics

\begin{tabular}{lc}
\hline \multicolumn{1}{c}{ Characteristic } & Value \\
\hline Age $(\mathrm{y})$ & $47 \pm 9.97$ \\
Sex & \\
$\quad$ Male & $13(59.1)$ \\
Female & $9(40.9)$ \\
Height $(\mathrm{cm})$ & $166.5 \pm 8.42$ \\
Weight $(\mathrm{kg})$ & $73.34 \pm 12.82$ \\
Body mass index $\left(\mathrm{kg} / \mathrm{m}^{2}\right)$ & $26.45 \pm 4.24$ \\
Ethnicity & \\
Chinese & $14(63.6)$ \\
Indian & $4(18.2)$ \\
Malay & $2(9.1)$ \\
Other & $2(9.1)$ \\
Affected knee & \\
Left & $9(40.9)$ \\
Right & $13(59.1)$ \\
Concurrent injuries & \\
Osteoarthritis & $13(59.1)$ \\
Meniscal tear & $10(45.5)$ \\
MCL/LCL sprain & $7(31.8)$ \\
ACL sprain & $4(18.2)$ \\
\hline
\end{tabular}

Data are mean \pm standard deviation or $\mathrm{n}(\%)$. Abbreviations: ACL, anterior cruciate ligament; LCL, lateral collateral ligament; MCL, medial collateral ligament.
Table 3. Characteristics of articular cartilage lesions

\begin{tabular}{lccc}
\hline Anatomic Location & Frequency & Size $(\mathrm{mm})$ & $\begin{array}{c}\text { Mean ICRS } \\
\text { Grade }\end{array}$ \\
\hline $\begin{array}{l}\text { Medial femoral } \\
\text { condyle }\end{array}$ & & & \\
1 & 0 & 0 & 0 \\
2 & 3 & 8.7 & 3 \\
3 & 0 & 0 & 0 \\
4 & 1 & 7 & 4 \\
5 & 6 & 15.8 & 4 \\
6 & 2 & 13 & 4 \\
7 & 0 & 0 & 0 \\
8 & 3 & 11.7 & 4 \\
9 & 0 & 0 & 0 \\
Lateral femoral & & & \\
condyle & & & \\
1 & 0 & 0 & 0 \\
2 & 0 & 0 & 0 \\
3 & 0 & 0 & 0 \\
4 & 0 & 0 & 3 \\
5 & 4 & 8.75 & 0 \\
6 & 0 & 0 & 0 \\
7 & 0 & 0 & 0 \\
8 & 0 & 0 & 0 \\
9 & 0 & 0 & 4 \\
Femoral & & & \\
trochlea & & 10 & 4 \\
1 & & 13 & \\
2 & 4 & & \\
3 & 5 & & \\
\hline
\end{tabular}

Based on preoperative imaging and intra-operative findings, articular cartilage lesions are described according to their location, size, and mean International Cartilage Repair Society (ICRS) grade. Specific locations of the lesions are detailed in Figure 1A-C.

documented to have complications or reoperations during the 2-year follow-up period.

\section{Discussion}

The results of this study indicate that patients who underwent microfracture with the Hyalofast scaffold improved their IKDC scores significantly in the short term at 6 months and midterm at 2 years postoperatively. As the minimum clinically important difference for IKDC scores is 11.5 , these results suggest that the improvements are both statistically and clinically significant. ${ }^{19,20}$ The overall clinical result of a mean $97 \%$ improvement in IKDC scores at the 2-year follow-up suggests comparable or even higher results than what is reported for microfracture alone, which ranges from $70 \%$ to $95 \% .^{21-23}$ These results suggest that microfracture using a cell-free hyaluron-based scaffold for small articular cartilage lesions may be as effective as, or even more effective than, a single-stage option for patients with articular cartilage defects of the knee. Potential causes for improved postoperative scores compared with microfracture alone include greater mechanical stability, improved defect filling, concentrating effect conferred by the Hyalofast matrix, 
or all-arthroscopic approach, but further studies with postoperative arthroscopy, imaging, or histological analysis are likely required to confirm such conjecture.

Additionally, the imaging results analyzed in this study indicate that the vast majority of articular cartilage defects occur over the center of the medial femoral condyles. This finding is consistent with the literature, which suggests that the location may correspond with the region of highest pressure on the weightbearing surface. ${ }^{18,24,25}$ Whereas previous studies have focused on describing cartilage lesions by the Outerbridge, ICRS, or Bauer-Jackson classifications, there is limited existing literature on the specific location of the articular cartilage lesions, and the results of this study provide greater detail on the distribution of articular cartilage lesions in the knee.

Previous studies examining outcomes after microfracture correlated age $>30$ years, BMI $>30 \mathrm{~kg} / \mathrm{m}^{2}$, and longer preoperative duration of symptoms with worse outcomes postoperatively. ${ }^{21}$ Furthermore, multiple long-term studies indicate that although short and medium term outcomes are excellent with microfracture, results worsen with time after the 2-year follow-up period, which is thought to be attributed to inferior quality of the regenerated cartilage, making it vulnerable to increased wear. ${ }^{26-28}$ As an early-stage study with limited patients and a 2-year follow-up period, our study was not able to comment on predictors for outcome and longer-term outcomes. Subsequent studies will likely aim to assess over longer periods of follow-up with greater patient numbers to determine how robust postoperative outcomes of allarthroscopic AMIC using cell-free biological scaffolds are in the long term and whether age, BMI, and preoperative duration of symptoms are similarly correlated with poorer postoperative outcomes.

Future studies should seek to characterize how AMIC using hyaluron-based scaffolds affects cartilage regeneration via microfracture with postoperative arthroscopy, repeat imaging, or histological studies. It may also be useful to characterize the specific biomechanical properties of the clot formed using the hyaluron-based scaffold in comparison to a standard microfracture procedure. Comparing AMIC with other established treatments for articular cartilage lesions, such as osteochondral grafting or osteochondral transfer systems, in terms of clinical outcome, cost, and patient satisfaction will be useful in defining treatment algorithms. Additionally, it may be useful to compare the outcomes from an all-arthroscopic approach to AMIC with those requiring a mini-arthrotomy or larger incision, particularly with regard to wound-related complications and postoperative outcomes. Lastly, because articular cartilage lesions of the knee predispose to early osteoarthritis, long-term clinical outcomes data would be useful in assessing AMIC's long-term efficacy.

\section{Limitations}

The main limitations of the current study were the retrospective evaluation of prospectively collected data, as well as the limited number of patients recruited into the study. Because of patients lost to follow-up or excluded owing to incomplete data, only $57.9 \%$ of patients who underwent the procedure were included for analysis, and only 1 outcome metric, the IKDC score, was used in evaluating patients. Preoperative duration of symptoms was not documented. In view of cost to patients, performing postoperative MRI scans and arthroscopy were not routinely performed and could not be used to assess for cartilage healing. As the study was designed as a preliminary analysis of a new technique for the AMIC procedure, the power of the study was not calculated and may not be adequate due to limited number of patients.

\section{Conclusion}

An all-arthroscopic technique for single-stage autologous matrix-induced chondrogenesis demonstrates significant early clinical improvement for the treatment of articular cartilage lesions of the knee.

\section{Acknowledgments}

The authors thank Dr. Lee Man Xin for assisting with data collection and analysis.

\section{References}

1. Magnussen RA, Dunn WR, Carey JL, Spindler KP. Treatment of focal articular cartilage defects in the knee. Clin Orthop Rel Res 2008;466:952-962.

2. Bark S, Piontek T, Behrens P, Mkalaluh S, Varoga D, Gille J. Enhanced microfracture techniques in cartilage knee surgery: fact or fiction? World J Orthop 2014;5:444.

3. Sofu H, Kockara N, Oner A, Camurcu Y, Issin A, Sahin V. Results of hyaluronic acid-based cell-free scaffold application in combination with microfracture for the treatment of osteochondral lesions of the knee: 2-year comparative study. Arthroscopy 2017;33:209-216.

4. Zanasi S, Ventura C. One step tissue engineering for cartilage reconstruction in severe osteoarthritis of the knee and ankle: a comprehensive review of the technique resorting to isolated BMAC or ADSCS and their last combination. NanoWorld J 2017;3:S9-S15.

5. Gobbi A, Whyte GP. One-stage cartilage repair using a hyaluronic acid-based scaffold with activated bone marrow-derived mesenchymal stem cells compared with microfracture: five-year follow-up. Am J Sports Med 2016;44:2846-2854.

6. Verdonk P, Dhollander A, Almqvist K, Verdonk R, Victor J. Treatment of osteochondral lesions in the knee using a cell-free scaffold. Bone Joint J 2015;97:318-323.

7. Pridie K. A method of resurfacing osteoarthric knee joints. J Bone Joint Surg Br 1959;3:618-619.

8. Steadman JR, Briggs KK, Rodrigo JJ, Kocher MS, Gill TJ, Rodkey WG. Outcomes of microfracture for traumatic 
chondral defects of the knee: average 11-year follow-up. Arthroscopy 2003;19:477-484.

9. Hunziker EB. Articular cartilage repair: basic science and clinical progress. A review of the current status and prospects. Osteoarthritis Cartilage 2002;10:432-463.

10. Mankin HJ. The response of articular cartilage to mechanical injury. J Bone Joint Surg Am 1982;64:460-466.

11. Benthien JP, Behrens P. Autologous matrix-induced chondrogenesis (AMIC). A one-step procedure for retropatellar articular resurfacing. Acta Orthop Belg 2010;76:260.

12. Lee YHD, Suzer F, Thermann H. Autologous matrixinduced chondrogenesis in the knee: a review. Cartilage 2014;5:145-153.

13. Gobbi A, Nunag P, Malinowski K. Treatment of full thickness chondral lesions of the knee with microfracture in a group of athletes. Knee Surg Sports Traumatol Arthrosc 2005;13:213-221.

14. Kramer J, Böhrnsen F, Lindner U, Behrens P, Schlenke P, Rohwedel J. In vivo matrix-guided human mesenchymal stem cells. Cell Mol Life Sci 2006;63:616.

15. Gobbi A, Karnatzikos G, Scotti C, Mahajan V, Mazzucco L, Grigolo B. One-step cartilage repair with bone marrow aspirate concentrated cells and collagen matrix in fullthickness knee cartilage lesions: results at 2-year followup. Cartilage 2011 ; 2:286-299.

16. Higgins LD, Taylor MK, Park D, Ghodadra N, Marchant M, Pietrobon R, Cook C, International Knee Documentation Committee. Reliability and validity of the International Knee Documentation Committee (IKDC) subjective knee form. Joint Bone Spine 2007;74:594-599.

17. da Cunha CB, Andrade R, Veloso TR, Learmonth DA, Espregueira-Mendes J, Sousa RA. Enhanced microfracture using acellular scaffolds improves results after treatment of symptomatic focal grade III/IV knee cartilage lesions but current clinical evidence does not allow unequivocal recommendation. Knee Surg Sports Traumatol Arthrosc 2020:1-13.

18. Årøen A, Løken S, Heir S, et al. Articular cartilage lesions in 993 consecutive knee arthroscopies. Am J Sports Med 2004;32:211-215.

19. Valier AR, Lam KC. Beyond the basics of clinical outcomes assessment: selecting appropriate patient-rated outcomes instruments for patient care. Athletic Training Ed J 2015;10:91-100.

20. Collins NJ, Misra D, Felson DT, Crossley KM, Roos EM. Measures of knee function: International Knee Documentation Committee (IKDC) Subjective Knee Evaluation Form, Knee Injury and Osteoarthritis Outcome Score (KOOS), Knee Injury and Osteoarthritis Outcome Score Physical Function Short Form (KOOS-PS), Knee Outcome Survey Activities of Daily Living Scale (KOS-ADL), Lysholm Knee Scoring Scale, Oxford Knee Score (OKS), Western Ontario and McMaster Universities Osteoarthritis Index (WOMAC), Activity Rating Scale (ARS), and Tegner Activity Score (TAS). Arthritis Care Res 2011;63: S208-S228.

21. Mithoefer K, WILLIAMS RJ III, Warren RF, et al. The microfracture technique for the treatment of articular cartilage lesions in the knee: a prospective cohort study. J Bone Joint Surg Am 2005;87:1911-1920.

22. Steadman J. Microfracture technique for full-thickness chondral defects: technique and clinical results. Operative Tech Orthop 1997;7:294-299.

23. Steadman JR, Karas SG, Schlegel TF. The microfracture technique in the treatment of full-thickness chondral lesions of the knee in National Football League players. J Knee Surg 2003;16:83-86.

24. Hjelle K, Solheim E, Strand T, Muri R, Brittberg M. Articular cartilage defects in 1,000 knee arthroscopies. Arthroscopy 2002;18:730-734.

25. Curl WW, Krome J, Gordon ES, Rushing J, Smith BP, Poehling GG. Cartilage injuries: a review of 31,516 knee arthroscopies. Arthroscopy 1997;13:456-460.

26. Bae DK, Song SJ, Yoon KH, Heo DB, Kim TJ. Survival analysis of microfracture in the osteoarthritic knee-minimum 10-year follow-up. Arthroscopy 2013;29: 244-250.

27. Goyal D, Keyhani S, Lee EH, Hui JHP. Evidence-based status of microfracture technique: a systematic review of level I and II studies. Arthroscopy 2013;29:1579-1588.

28. Solheim E, Hegna J, Inderhaug E, Øyen J, Harlem T, Strand T. Results at 10-14 years after microfracture treatment of articular cartilage defects in the knee. Knee Surg Sports Traumatol Arthrosc 2016;24:1587-1593. 